\title{
Direct observation of bulk second-harmonic generation inside a glass slide with tightly focused optical fields
}

\author{
Xianghui Wang, ${ }^{1,2}$ Shima Fardad, ${ }^{1}$ Susobhan Das, ${ }^{1}$ Alessandro Salandrino, ${ }^{1}$ and Rongqing Hui ${ }^{1, *}$ \\ ${ }^{1}$ Department Electrical Engineering \& Computer Science, University of Kansas, Lawrence, Kansas 66045, USA \\ ${ }^{2}$ Institute of Modern Optics, Nankai University, Key Laboratory of Optical Information Science and Technology, \\ Ministry of Education, Tianjin 300071, China
}

(Received 24 August 2015; revised manuscript received 29 March 2016; published 18 April 2016)

\begin{abstract}
Bulk second-harmonic generation (SHG) inside glass slides is directly detected unambiguously without interference from surface contributions. This is enabled by tightly focused and highly localized ultrashort laser pulses. The theoretical calculations based on vector diffraction theory and the phenomenological model of SHG inside centrosymmetric materials agree well with the measured far-field SHG radiation patterns for different polarization states of the fundamental beam. The results indicate that the observed bulk SHG is predominantly related to the bulk parameter $\delta^{\prime}$ and originates from the three-dimensional field gradient in the focal region.
\end{abstract}

DOI: 10.1103/PhysRevB.93.161109

Under the electric dipole approximation of light-matter interaction, second-harmonic generation (SHG) is usually considered forbidden in bulk centrosymmetric materials. Dipolar SHG is allowed through a surface contribution arising at the interface between centrosymmetric materials where centrosymmetry is broken $[1,2]$. However, when higher-order multipole interactions $[3,4]$, such as magnetic-dipole and electric-quadrupole interactions, are taken into account, SHG can occur even inside bulk centrosymmetric materials, which was observed in several experiments [5-8]. When SHG is used as a surface or interface probe, it is essential to separate bulk contributions from the measured SH signal and verify that such multipolar contributions are actually negligible [9]. In addition, as suggested recently, strong multipole interactions may bypass the noncentrosymmetry requirement and promote applications of new nonlinear materials $[10,11]$. Therefore, a thorough understanding of the physical mechanisms behind bulk SHG from bulk centrosymmetric materials is fundamental for many practical applications utilizing SHG [12].

Various attempts have been made to characterize surface and bulk contributions to SHG $[7,8,13,14]$. For example, the SHG based on two noncollinear fundamental beams $[7,8]$ has been widely used to study surface and bulk contributions in a quantitative way. However, up to now, the separation of surface and bulk contributions is still known to be a fundamental difficulty in the field of SHG from centrosymmetric materials. Since two-beam overlap in a spatial region is usually several millimeters long [8], the excitation field is not highly localized. Furthermore, as the SH signal is often too weak to detect when the interaction volume is deep inside the bulk material, the excitation region is often chosen to be near the surface in order to maximize signal intensity [15]. Thus the surface dipolar response cannot be completely avoided, which often perturbs the bulk response. This contributes to the wide variation of results reported in the literature. So far, the mechanism of bulk SHG inside centrosymmetric materials still remains unclear.

Bulk SHG has been previously observed in centrosymmetric gaseous materials $[16,17]$ with focused laser pulses, where

*Corresponding author: rhui@ku.edu
SHG was attributed to the ponderomotive force caused by the transverse field gradient. To the best of our knowledge, the generation of bulk $\mathrm{SH}$ inside solid materials such as glass using a tightly focused excitation field has not been reported. Here, we demonstrate bulk SHG inside several types of glass slides using a simple experimental setup based on a single tightly focused, high-intensity femtosecond laser beam. Owing to the highly localized excitation field, when the focal spot is completely moved inside the bulk of a glass slide, the surface contribution can be effectively eliminated and only the bulk contribution related to the field gradient is present. This allows the investigation and clarification of bulk SHG mechanisms without interference from other sources, which was not possible with previous techniques. The theoretical results of SHG radiation patterns agree well with experimental observations. Those results indicate that the detected bulk SHG is predominantly created by $\left[\mathbf{E}^{(\omega)}(\mathbf{r}) \cdot \nabla\right] \mathbf{E}^{(\omega)}(\mathbf{r})$, which arises from the three-dimensional (3D) gradient of the tightly focused excitation field $\mathbf{E}^{(\omega)}(\mathbf{r})$ at the fundamental frequency $\boldsymbol{\omega}$.

For an isotropic material, the bulk response can be phenomenologically described by a nonlocal bulk polarization $\mathbf{P}_{\text {bulk }}^{(2 \omega)}(\mathbf{r})[7,8,18,19]$,

$$
\begin{aligned}
\mathbf{P}_{\text {bulk }}^{(2 \omega)}(\mathbf{r})= & \beta \mathbf{E}^{(\omega)}(\mathbf{r})\left[\nabla \cdot \mathbf{E}^{(\omega)}(\mathbf{r})\right]+\gamma \nabla\left[\mathbf{E}^{(\omega)}(\mathbf{r}) \cdot \mathbf{E}^{(\omega)}(\mathbf{r})\right] \\
& +\delta^{\prime}\left[\mathbf{E}^{(\omega)}(\mathbf{r}) \cdot \nabla\right] \mathbf{E}^{(\omega)}(\mathbf{r})
\end{aligned}
$$

where $\beta, \gamma$, and $\delta^{\prime}$ are material parameters which depend on the magnetic-dipole and electric-quadruple tensors of the isotropic centrosymmetric material. The first term on the right-hand side of Eq. (1) vanishes for a homogeneous medium in which $\boldsymbol{\nabla} \cdot \mathbf{E}(\mathbf{r})=\mathbf{0}$. In traditional measurement techniques, the contribution from $\gamma$ is usually included in an effective surface response due to the fact that it always behaves as the surface contribution and cannot be separated from the surface response [9], which is also the fundamental difficulty in the use of SHG as a strictly surface probe. When there is only a single plane wave (PW) in the bulk of the centrosymmetric material, the parameter $\delta^{\prime}$ cannot contribute to bulk SHG. However, if a strong field gradient occurs, e.g., in the case of a two-beam scheme or focused field excitation, the contribution from $\delta^{\prime}$ will be present. 


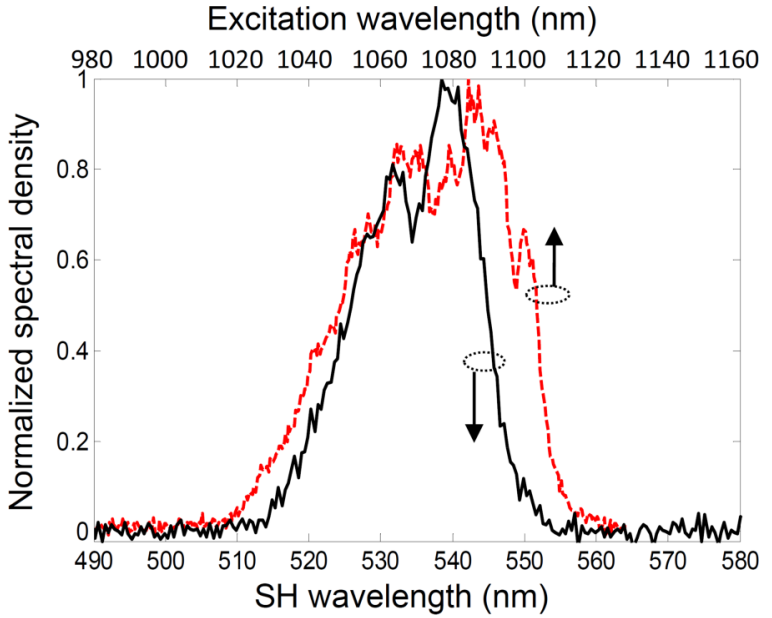

FIG. 1. Spectra of the fundamental (red dashed line) and bulk generated SH (black solid line) beams, recorded by the spectrometer.

In our experiment, a femtosecond fiber laser (Fidelity, Coherent, Inc.) was used as the fundamental excitation beam for SHG. The central wavelength of the laser is $1075 \mathrm{~nm}$ with a $78 \mathrm{MHz}$ pulse repetition rate and an approximately $60 \mathrm{fs}$ pulse duration. A polarizer was placed at the laser output to guarantee the linear polarization, while the polarization state of the fundamental excitation beam was varied by a rotating quarter-wave plate. The beam was focused by an infinitycorrected dry objective lens (LCPLN-IR, 100X, numerical aperture 0.85 , Olympus Corp.) with a long working distance of $1.2 \mathrm{~mm}$ [20] into glass slides. The transverse and axial lengths of the focused field inside the glass slide formed by the objective lens are approximately 1.0 and $3.0 \mu \mathrm{m}$, respectively (see Supplemental Material [21]). The position of the glass slide can be displaced along the beam propagation direction by a motorized translation stage with a $1 \mu \mathrm{m}$ step size. Thus the excitation focal point can be moved from the front surface to the back surface through the bulk of the glass. After beam propagation inside the sample, it is collected and collimated by another identical objective lens. A short-pass filter is used to remove the fundamental beam, and the $\mathrm{SH}$ signal is detected by a spectrometer (Maya2000 Pro, Ocean Optics, Inc.). In addition, the $\mathrm{SH}$ emission patterns corresponding to different excitation conditions are recorded by a video camera.

Both SHG in the two-beam scheme [8] and third-harmonic generation (THG) under focused field excitation [22] from BK7 glass have been previously studied, SHG based on a focused excitation field inside the bulk of a glass slide has not been reported. We first used a borosilicate glass cover slide as the sample, and the excitation beam was focused inside the bulk of the glass slide. SH at a central wavelength of $537 \mathrm{~nm}$ was measured by the spectrometer, as shown in Fig. 1. The spectrum of the fundamental excitation beam with a central wavelength of $1075 \mathrm{~nm}$ is also displayed for comparison. Note that under this excitation condition with the focal point inside the glass sample, THG was too weak to be measured.

A more systemic measurement was then performed by scanning the sample position along the beam propagation direction, so that the focal point could be moved into and out of the sample continuously. Figure 2 shows the normalized

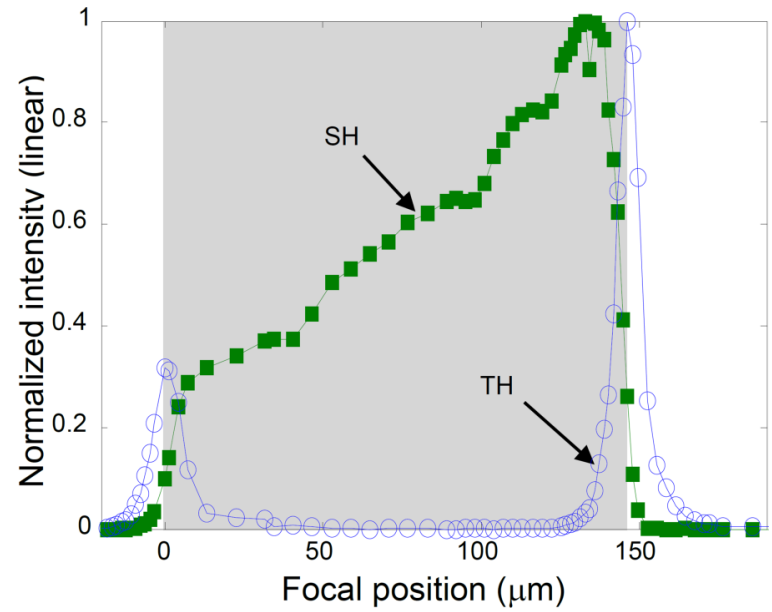

FIG. 2. Measured intensities of SHG (solid squares) and THG (open circles) as the function of focal position inside a borosilicate glass slide.

intensities of SH and third-harmonic (TH) signals, respectively, measured by the spectrometer as the function of the focal position inside the sample. The shaded region indicates the cross section of the glass slide. Considering the refractive index $n=1.507$ of borosilicate at $1075 \mathrm{~nm}$ excitation wavelength, the glass thickness of $146 \mu \mathrm{m}$ corresponds to a sample traveling distance of $97 \mu \mathrm{m}$ from one surface to the other. In the process of moving the excitation focal point from air into the glass slide, a strong TH signal was observed when the focal point is at the air/glass interface. This agrees with the previously reported strong THG at the interface between different materials $[22,23]$. No TH was measurable in the bulk of the glass due to the limitation of the coherence length with respect to the Gouy phase shift [24]. On the contrary, a relatively low $\mathrm{SH}$ signal was measured at the glass surface. A significantly increased SH signal was observed only when the focal point was moved completely into the bulk of the glass. In fact, the color change from blue (TH at $359 \mathrm{~nm}$ ) to green ( $\mathrm{SH}$ at $538 \mathrm{~nm}$ ) could be clearly seen even with the naked eye by placing white paper in front of the spectrometer and slowly scanning the focal position from air into the glass slide. Figure 2 also shows that the SH intensity gradually increases when moving the excitation focal point further into the glass until it reaches the maximum near the second surface. In another experiment based on the similar setup, $z$-scan measurements of SHG were performed at different pump power levels and different aberration-correction settings of the objective lens (see Figs. S4 and S5 [21]). It becomes apparent that aberration correction of the objective lens has played a key role in the increase of SHG when the focal point was moved deeper inside the glass slide.

Generally, high-order multipole interactions are considered much weaker than surface electric-dipole interactions. However, the results shown in Fig. 2 reveal a much stronger bulk SHG inside the centrosymmetric bulk. The sharp peak of THG at each surface of the sample indicates that the Rayleigh length of the focused optical field is much shorter than the thickness of the glass slide, and the SHG observed inside the glass sample in our experiment was created predominately 


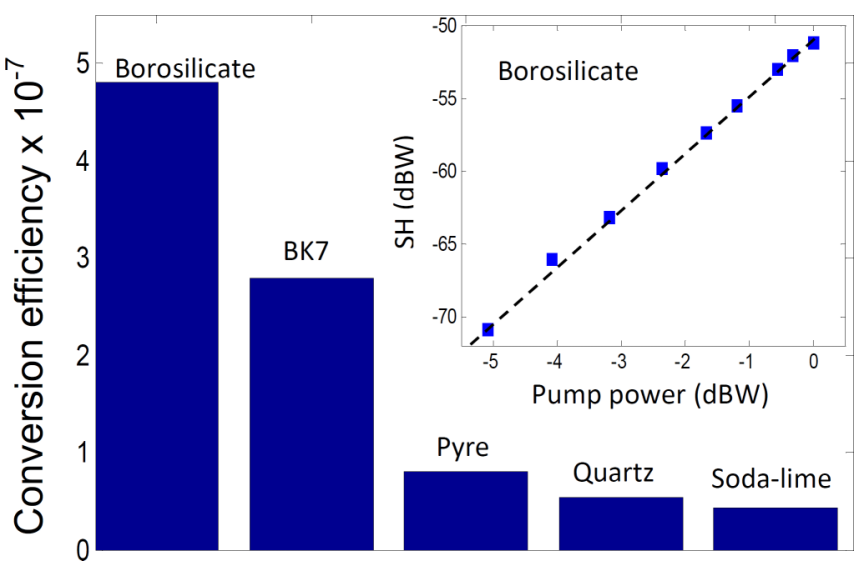

FIG. 3. Power conversion efficiency of bulk SHG from borosilicate, BK7, Pyrex, and soda lime glass samples.

from the bulk contribution. Although the surface response can be generated by the longitudinal component of the focused field, this component is usually weak, and more importantly the maximum of this longitudinal component only exists at the focal plane. Therefore, when the focus is moved away from the surface of the glass slide, the magnitude of this longitudinal component gradually diminishes, and is eventually suppressed completely when the focal point is deep inside the glass slide. It should be pointed out that no noticeable bulk SHG was observed inside the bulk glass in previous THG experiments in which glass slides were also excited by focused excitation fields $[22,23]$. This was due to the relatively small numerical aperture of the focusing objective lens used in those experiments, which resulted in a greatly reduced field gradient at the focal region, and thus much lower SHG efficiency inside the bulk [21].

Several other types of glass samples have also been tested, including BK7, Pyrex, quartz, and soda lime. The thickness of the borosilicate glass sample is $146 \mu \mathrm{m}$, while other glass samples are all approximately $1 \mathrm{~mm}$ thick. Nevertheless, the measured SHG and THG are characteristically similar to those described in Fig. 2, including two peaks of THG at the surfaces and increasing SH intensity along the $z$-scan direction. The power conversion efficiency, defined as the power ratio between the $\mathrm{SH}$ output and the fundamental input, was measured for each sample with $0.5 \mathrm{~W}$ excitation pump power. This is equivalent to a pulse peak power of approximately $1 \mathrm{MW}$, which is slightly lower than the self-focusing threshold power of 1.5 MW for the glass [25]. Considering the $1 \mu \mathrm{m}$ focal spot diameter, the power density of $1.4 \times 10^{17} \mathrm{~W} / \mathrm{m}^{2}$ is also approximately half of the breakdown power density of the glass [25,26]. For fair comparison, the focal point was positioned $137 \mu \mathrm{m}$ inside the sample (measured from the front surface) for all samples. As shown in Fig. 3, the power conversion efficiency due to bulk SHG ranges from $0.5 \times 10^{-7}$ to $5 \times 10^{-7}$ for these glass samples. The inset of Fig. 3 shows the pump power dependency of bulk SHG inside the borosilicate glass in $\mathrm{dB} / \mathrm{dB}$ scale. The slope of approximately $4 \mathrm{~dB} / \mathrm{dB}$ shown in Fig. 3 indicates a strong impact of self-focusing in which the power density increases with the square of the pump power because of the size reduction of the focal point [21].

In order to better understand the basic mechanism of bulk SHG in glass samples, the impact of the state of polarization (SOP) of the incident $\mathrm{PW}$ on the $\mathrm{SH}$ radiation pattern was investigated. By rotating the quarter-wave plate, the SOP of the pump wave can be progressively changed from linear to circular, as illustrated in the first row of Fig. 4. $\varphi$ represents the angle between the polarization direction of the incident PW and the optical axis of the quarter-wave plate. The SHG radiation pattern corresponding to each SOP of the incident PW can then be recorded by a camera as shown by the second row of Fig. 4. The third and fourth rows of Fig. 4 show the results of theoretical calculations based on the contributions proportional to the parameters $\delta^{\prime}$ and $\gamma$, respectively. With $\varphi=0^{\circ}$, the incident $\mathrm{PW}$ is $x$-linearly polarized. The radiation pattern of SH exhibits a two-lobe structure, and the orientation of those two lobes is perpendicular to the $x$ direction. It is worth noting that this orientation is $90^{\circ}$ apart from the previously reported results $[17,27]$, and this difference may help elucidate the fundamental mechanisms behind bulk SHG of different conditions. In our experiment, the bulk contribution primarily originates from the $3 \mathrm{D}$ field gradient, while the previously reported SHG [17,27] was predominately resulted from the transverse field gradient. This explanation was validated by theoretical analysis. Indeed, in the numerical modeling, by setting the field components in the $z$ direction to zero, the $\mathrm{SH}$ radiation pattern would rotate by $90^{\circ}$ [21]. With the increase of $\varphi$, the $\mathrm{SH}$ radiation maintains its two-lobe pattern, while the orientation gradually rotates. However, as $\varphi$ approaches $45^{\circ}$ (circular polarization) the radiation pattern quickly becomes doughnut shaped. For all of the $\varphi$ values investigated here, theoretical calculations based only on the parameter $\delta^{\prime}$ produce $\mathrm{SH}$ radiation patterns which match the experimental results well. Meanwhile, the calculated radiation patterns corresponding to $\gamma$ exhibit a constant doughnut shape independent of the SOP of the incident PW. The obvious $\varphi$ angle dependence of the measured $\mathrm{SH}$ radiation pattern clearly implies that $\delta^{\prime}$ is the dominant contribution to the bulk SHG. The minor difference between the measured and calculated $\mathrm{SH}$ radiation patterns can be attributed to the residual contribution from the parameter $\gamma$. In Ref. [8], a quantitative tensor analysis of the effective dipolar surface nonlinearity and multipolar bulk nonlinearity was performed using the two-beam scheme. It was found that the ratio between the two bulk parameters was approximately $\gamma / \delta^{\prime} \approx-0.5$. Apparently, radiation patterns calculated with this ratio, as shown in the last row of Fig. 4, cannot reproduce our experimental observations. Instead, the comparison between our experimental and theoretical results suggests that the contribution from $\delta^{\prime}$ is much stronger than that predicted in Ref. [8], and the magnitude of the ratio of $\left|\gamma / \delta^{\prime}\right|$ should be lower than 0.1 in SHG measured with a tightly focused excitation field. This conclusion was reached from our unique experimental setup based on a tightly focused excitation beam which eliminated the interference from surface response.

In conclusion, a bulk SH response inside glass slides was directly detected without interference from the surface contribution. When the incident PW was not circularly polarized, a two-lobe-shaped $\mathrm{SH}$ radiation pattern was observed with an 

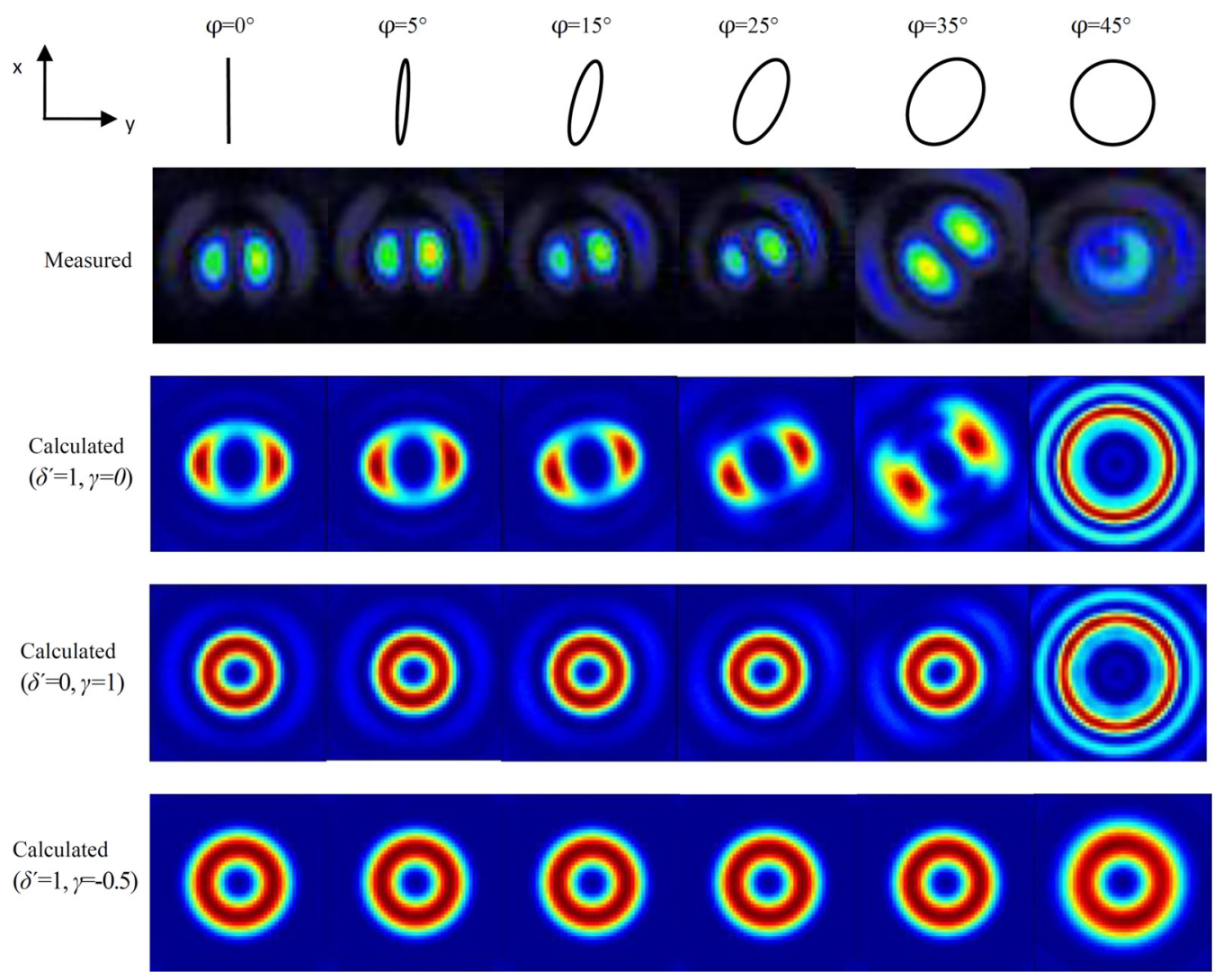

FIG. 4. SH radiation patterns for different $\varphi$ values. Values used for $\varphi$ and their corresponding polarization states of the incident PW (first row), the experimentally measured patterns (second row), theoretical calculations for $\delta^{\prime}=1$ and $\gamma=0$ (third row), $\delta^{\prime}=0$ and $\gamma=1$ (fourth row), and $\delta^{\prime}=1$ and $\gamma=-0.5$ (fifth row).

orientation $90^{\circ}$ different from the previously reported results. This difference is attributed to the strong 3D gradient of the excitation field used in our experiment. The comparison between theoretical and experimental results for $\mathrm{SH}$ radiation patterns with different SOPs of the incident PW indicates that the parameter $\delta^{\prime}$ was the dominant contribution to bulk SHG, which is much stronger than the previous estimation.

The work of X.W. was partially supported by the National Natural Science Foundation of China (Grant No. 61378005).
[1] Y. R. Shen, Nature (London) 337, 519 (1989).

[2] R. W. Boyd, Nonlinear Optics (Academic, New York, 1992).

[3] J. A. Armstrong, N. Bloembergen, J. Ducuing, and P. S. Pershan, Phys. Rev. 127, 1918 (1962).

[4] P. Guyot-Sionnest and Y. R. Shen, Phys. Rev. B 38, 7985 (1988).

[5] M. Fiebig, D. Fröhlich, Th. Lottermoser, V. V. Pavlov, R. V. Pisarev, and H.-J. Weber, Phys. Rev. Lett. 87, 137202 (2001).

[6] P. Figliozzi, L. Sun, Y. Jiang, N. Matlis, B. Mattern, M. C. Downer, S. P. Withrow, C. W. White, W. L. Mochán, and B. S. Mendoza, Phys. Rev. Lett. 94, 047401 (2005).

[7] S. Cattaneo and M. Kauranen, Phys. Rev. B 72, 033412 (2005).

[8] F. J. Rodríguez, F. X. Wang, B. K. Canfield, S. Cattaneo, and M. Kauranen, Opt. Express 15, 8695 (2007).

[9] J. E. Sipe, V. Mizrahi, and G. I. Stegeman, Phys. Rev. B 35, 9091 (1987).

[10] M. W. Klein, C. Enkrich, M. Wegener, and S. Linden, Science 313, 502 (2006).

[11] D. J. Cho, F. Wang, X. Zhang, and Y. R. Shen, Phys. Rev. B 78, 121101 (2008).
[12] M. Kauranen and A. V. Zayats, Nat. Photonics 6, 737 (2012).

[13] G. Bachelier, J. Butet, I. Russier-Antoine, C. Jonin, E. Benichou, and P.-F. Brevet, Phys. Rev. B 82, 235403 (2010).

[14] F. X. Wang, F. J. Rodríguez, W. M. Albers, R. Ahorinta, J. E. Sipe, and M. Kauranen, Phys. Rev. B 80, 233402 (2009).

[15] S. Cattaneo, M. Siltanen, F. X. Wang, and M. Kauranen, Opt. Express 13, 9714 (2005).

[16] G. H. Li, J. L. Ni, H. Q. Xie, B. Zeng, J. P. Yao, W. Chu, H. S. Zhang, C. R. Jing, F. He, H. L. Xu, Y. Cheng, and Z. Z. Xu, Opt. Lett. 39, 961 (2014).

[17] M. Beresna, P. G. Kazansky, Y. Svirko, M. Barkauskas, and R. Danielius, Appl. Phys. Lett. 95, 121502 (2009).

[18] J. I. Dadap, J. Shan, and T. F. Heinz, J. Opt. Soc. Am. B 21, 1328 (2004).

[19] B. Koopmans, A. M. Janner, H. T. Jonkman, G. A. Sawatzky, and F. van der Woude, Phys. Rev. Lett. 71, 3569 (1993).

[20] http://www.olympus-ims.com/en/microscope/lmlcpln-ir/

[21] See Supplemental Material at http://link.aps.org/supplemental/ 10.1103/PhysRevB.93.161109 for details on the experimental 
setup, the analyses of $z$-scan measurements of glass slides, and the dependence of SH peak amplitude on pump power, the theoretical model of bulk SHG inside centrosymmetric material under tightly focused excitation, and related numerical simulations.

[22] E. C. Barbano, S. C. Zílio, and L. Misoguti, Opt. Lett. 38, 5165 (2013).
[23] T. Y. F. Tsang, Phys. Rev. A 52, 4116 (1995).

[24] J. X. Cheng and X. S. Xie, J. Opt. Soc. Am. B 19, 1604 (2002).

[25] C. B. Schaffer, A. Brodeur, J. F. García, and E. Mazur, Opt. Lett. 26, 93 (2001).

[26] A. Ben-Yakara and R. L. Byer, J. Appl. Phys. 96, 5316 (2004).

[27] M. G. Mandujano and J. A. Maytorena, Phys. Rev. A 88, 023811 (2013). 